\title{
A rare case of biventricular non-compaction cardiomyopathy associated with ventricular septal defect and atrial septal aneurysm
}

\author{
Maria Kordybach ${ }^{1}$, Mirosław Kowalski ${ }^{1}$, Łukasz Małek ${ }^{2,3}$, Piotr Hoffman ${ }^{1}$ \\ ${ }^{1}$ Department of Congenital Heart Disease, Institute of Cardiology, Warsaw, Poland \\ ${ }^{2}$ Department of Cardiology and Interventional Angiology, Institute of Cardiology, Warsaw, Poland \\ ${ }^{3}$ Department of Magnetic Resonance, Institute of Cardiology, Warsaw, Poland
}

A 35 year-old woman with a restrictive ventricular septal defect and an atrial septal aneurysm was admitted to our clinic. The patient reported mildly limited physical activity and palpitations. Transtho- racic echocardiography showed an enlarged left ventricle and left atrium. The size of the right heart cavities was normal. The structure of both ventricle walls revealed a compact epicardial layer and an

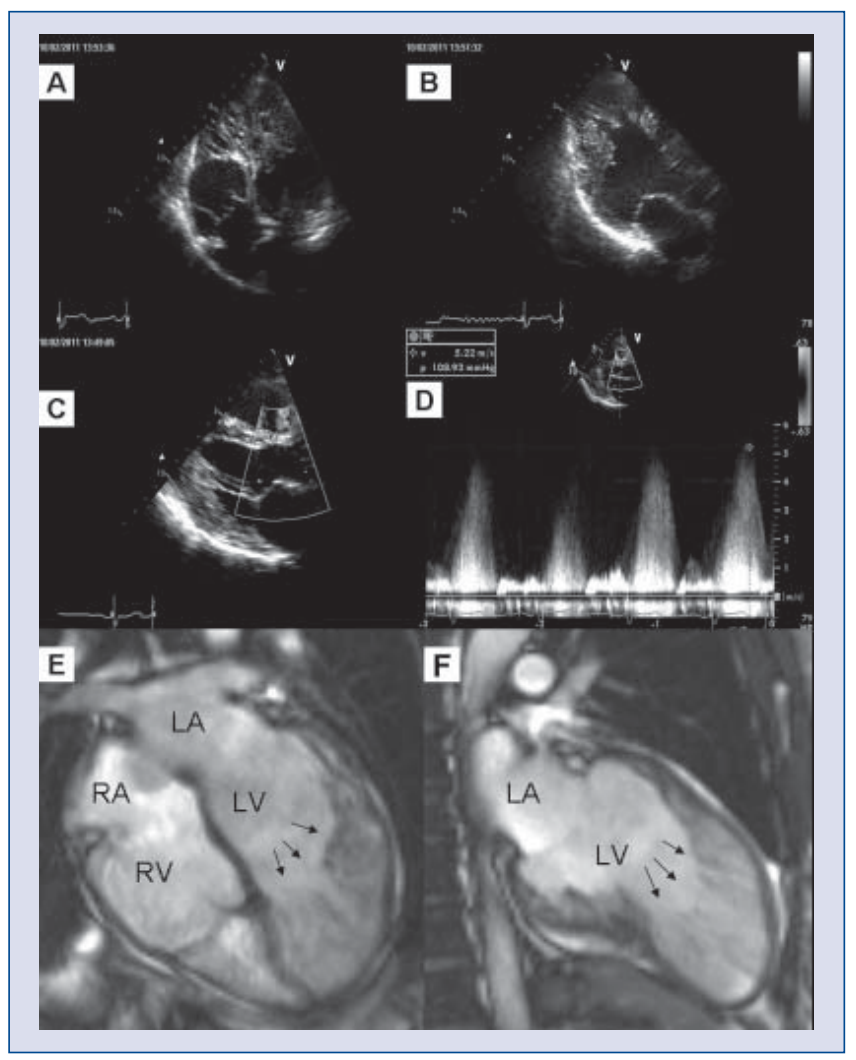

Figure 1. Echocardiography (A-D). Large trabecular meshwork and deep intertrabecular spaces of non-compacted myocardium in four- and three-chamber views (A, B). Small defect of interventricular septum (C) with left-to-right shunt and gradient of $110 \mathrm{~mm} \mathrm{Hg}$ (D). Cardiac magnetic resonance imaging (E, F). Cine balanced steady-state free precession images in end-diastole in four- and two-chamber views. The area of non-compacted myocardium is shown by black arrows; LA — left atrium; LV — left ventricle; RA — right atrium; RV — right ventricle.

Address for correspondence: Maria Kordybach, MD, Institute of Cardiology, Department of Congenital Heart Disease in Adults, ul. Alpejska 42, 04-628 Warsaw, Poland; tel: +48 22343 42 63, fax: +48 22343 45 38, e-mail: mkordybach@ikard.pl Received: 06.01.2012

Accepted: 11.01.2012 
endocardial layer consisting of a trabecular meshwork and deep intertrabecular spaces filled with Doppler color flow. The ejection fraction (EF) and peak systolic tissue velocity of basal septal segment were decreased (EF $30 \%$, s' $8 \mathrm{~cm} / \mathrm{s}$, respectively). There was a small defect of the membranous interventricular septum with left-to-right shunt and maximum left ventricle to right ventricle gradient of $110 \mathrm{~mm} \mathrm{Hg}$. In the atrial septum, there was an aneurysm without signs of shunt. Magnetic resonance imaging was performed to confirm the diagnosis. The examination proved the non-compaction cardiomyopathy (NCCM) with the ratio of non-compact/compact layer $>2.3: 1$ [1]. The left-to-right shunt was estimated for $10 \mathrm{~mL}$, which corresponded with insignificant pulmonary to systemic flow ratio (Qp:Qs 1.2:1). The 12-lead ECG Holter revealed more than 24,000 single ventricular extrasystoles. The patient was sent to the Electrophysiological Lab for ablation.

Ventricular non-compaction is a rare, unclassified cardiomyopathy. It results from lack of compaction of the loose myocardial meshwork during morphogenesis [2]. Heart failure, thromboembolism and ventricular arrhythmias are the major clinical manifestations [3]. NCCM can be seen as an isolated malformation or associated with an obstructive lesion of the outflow tracts [4]. Hence, it is rare when NCCM is linked with ventricular septal defect and an atrial septal aneurysm.

Conflict of interest: none declared

\section{References}

1. Petersen SE, Selvanayagam JB, Wiesmann F et al. Left ventricular non-compaction: Insights from cardiovascular magnetic resonance imaging. J Am Coll Cardiol, 2005; 46: 101-105.

2. Tatu-Chitoiu A, Bradisteanu S. A rare case of biventricular non-compaction associated with ventricular septal defect and descendent aortic stenosis in a young man. Eur J Echocardiogr, 2008; 9: 306-308.

3. Ozkutlu S, Ayabakan C, Celiker A, Elshershari H. Noncompaction of ventricular myocardium: A study of twelve patients. J Am Soc Echocardiogr, 2002; 15: 1523-1528.

4. Alehan D, Dogan OF. Right ventricular noncompaction in neonate with complex congenital heart disease. Cardiol Young, 2005; 15: 434-436. 